\title{
Obesidad en relación con el equilibrio dinámico de mujeres adultas mayores
}

\author{
Obesity in relationship with the dynamic balance of older adult women
}

*Jordan Hernández-Martínez

Hernández-Martínez, J. (2018). Obesidad en relación con el equilibrio dinámico de mujeres adultas mayores. Revista Ciencias de la Actividad Física UCM, N 19(2) julio-diciembre, 1-7. DOI: http://doi.org/10.29035/rcaf.19.2.5

\section{RESUMEN}

Objetivo: observar si la obesidad puede producir alteraciones en el equilibrio dinámico de mujeres adultas mayores. Método: se reclutaron treinta y cuatro mujeres adultas mayores con distinto estado nutricional (normo peso, sobrepeso y obesidad) en las cuales se midio en equilibrio dinámico mediante la prueba Timed up and Go. Resultados: se observaron diferencias estadísticamente significativas entre las mujeres adultas mayores normo peso en comparación a las con obesidad mostrado un mayor tiempo en la ejecución de la prueba de equilibrio dinámico. De igual manera, se observaron diferencias estadísticamente significativas entre las mujeres con sobrepeso y las normo peso, al igual que las mujeres con sobrepeso y obesidad. Conclusión: una acumulación excesiva de grasa corporal puede conducir a un mayor deterioro en el equilibrio dinámico de mujeres adultas mayores.

PALABRAS CLAVE

Grasa corporal, estado nutricional, estabilidad postural.

\begin{abstract}
Objective: observe if obesity can produce alterations in the dynamic balance of older adult women. Method: thirty-four older adult women with different nutritional status (normal weight, overweight and obese) were recruited in which their dynamic balance was measured using the Timed up and Go test. Results: Statistically significant differences were observed in older women in normal weight compared to obese in the execution of the dynamic balance test. Similarly, statistically significant differences were observed between overweight women and normal weight, as well as overweight and obese women. Conclusion: an excessive accumulation of body fat can lead to further deterioration in the dynamic balance of older adult women.
\end{abstract}

Key words

Fat body, nutritional status, postural stability.

* Profesor de Educación Física. Licenciado en Educación. Técnico Deportivo Universitario. Escuela de Terapia Ocupacional, Universidad Santo Tomás, Osorno, Chile. 


\section{INTRODUCCIÓN}

Es conocido que la obesidad es habitual en la vejez y que es un importante factor de riesgo modificable para los principales resultados adversos para la salud. Este aumento en la masa grasa se asocia con mayores niveles de factor inflamatorio relacionado con proteínaC-reactiva (PCR), inter leucina 6 (IL-6) e interleucina 1 receptor antagonista (IL-1 ra) en adultos mayores, de igual forma cambios en la distribución de la masa grasa aumentando la grasa visceral y almacenamiento de infiltración de lípidos en el músculo (Stenholm et al., 2008; Zamboni, Mazzali, Fantin, Rossi, \& Di Francesco, 2008). Este aumento en la masa grasa además de estar relacionado a alteraciones bioquímicas en el adulto mayor se relaciona con alteraciones en el funcionamiento de este grupo etario mostrando un mayor deterioro en la movilidad (Vincent, Vincent, \& Lamb, 2010), teniendo un mayor riesgo de caer (Mitchell, Lord, Harvey, \& Close, 2014), de discapacidad (Cawthon et al., 2011; Chen \& Guo, 2008) hospitalización, lo que termina afectando su independencia funcional, calidad de vida, salud y relación social (Wang et al., 2018).

Por su parte, el equilibrio también es una variable importante a considerar en el adulto mayor, siendo considerado un componente clave en varias actividades de la vida diaria, desde actividades simples como estar de pie hasta actividades más complejas como caminar mientras se habla o cambiar de dirección (Karimi \& Solomonidis, 2011). El equilibrio tiende a deteriora con la edad como resultado de alteraciones en los sistemas vestibular, visual, somatosensorial, musculoesquelético y nervioso central (da Silva Borges et al., 2014). Como consecuencia de esto, las personas muestran deficiencias en la estabilidad, el equilibrio y por lo tanto se exponen al riesgo de caer en situaciones tanto estáticas como dinámicas (Lord, Ward, Williams, \& Anstey, 1994; Desai, Goodman, Kapadia, Shay, \& Szturm, 2010). Se ha observado relación entre la obesidad y alteraciones en el equilibrio de adultos mayores (Teasdale et al., 2007). Si bien existe evidencia sobre la relación de estas variables, el propósi- to de este estudio es observar si existe relación entre una mayor grasa corporal con el equilibrio dinámico de mujeres adultas mayores.

\section{MÉTODO}

En un estudio descriptivo cuantitativo participaron treinta y cuatro mujeres del centro de adulto mayor correspondiente al sector de Rahue de la ciudad de Osorno. Este estudio fue aprobado por el comité de revisión científica y ética del departamento de ciencias de la actividad física y salud de la Universidad de los Lagos. Se incluyeron mujeres mayores de sesenta años de edad que no presentaran alguna patología cardiovascular, pulmonar o musculoesquelética lo cual se determinó mediante el cuestionario PAR-Q (The Physical Activity Readiness Questionnaire, pos sus siglas en Ingles). Las participantes que cumplieron con los criterios de inclusión y que accedieron a participar, firmaron un consentimiento informado, previa explicación de los potenciales riesgos y beneficios de su participación. El estudio se condujo de acuerdo a los principios éticos de la última versión de la Declaración de Helsinki 2013.

\section{Medidas antropométricas}

Se midió la estatura utilizando el plano de Frankfort en posición horizontal, con una cinta métrica (Bodymeter 206, SECA, Germany to $0.1 \mathrm{~cm}$ ) fijada a la pared. La grasa corporal se midió mediante bioimpedancia eléctrica registrando los datos en porcentaje. Se utilizó un bioimpedanciometro (InBody120, tetrapolar 8-point tactile electrodes system, model BPM040S12F07, Biospace, Inc., USA, to 0.1 $\mathrm{kg}$ ) según lo recomendado por Marfell, Olds, Stewart, \& Carter (2006). Los puntos de corte en el porcentaje de grasa fueron basados de acuerdo a la Organización Mundial de la Salud (OMS) normal (24-35,9\%) sobrepeso (36$41,9 \%)$ y obesidad ( $>42 \%)$ en mujeres adultas mayores (Gallagher et al., 2000). 


\section{Medida de equilibrio dinámico}

El equilibrio dinámico se midió mediante la prueba de Timed up and Go, este se aplicó según las recomendaciones previas (Posadillo \& Richardson, 1991). El sujeto en un gimnasio techado, el sujeto a la señal de "ya", debió pararse de una silla con reposabrazos, caminar por un pasillo de tres metros, girar y volver a sentarse a la silla. El sujeto ejecutó dos intentos de la prueba submáximos para familiarizarse con la prueba. Posterior a esto, se ejecutaron tres intentos máximos con una pausa mayor a noventa segundos registrando el mejor de los tres expresado en segundos. El tiempo será registrado mediante un cronómetro por un par evaluador externo.

\section{Análisis estadístico}

Los valores serán reportados como media \pm desviación estándar. Las características básicas y la clasificación de los grupos normo peso, sobrepeso y obesidad según puntos de corte establecidos por porcentaje de grasa corporal se presentan en la Tabla 1. Las pruebas de Shapiro-Wilk se aplicó para determinar la normalidad y Levene para determinar la homogeneidad de varianza, los datos arrojaron valores significativos para todos los datos. Para determinar los efectos de las condiciones en el equilibrio dinámico, las diferencias medias absolutas entre las condiciones de porcentaje de grasa corporal se compararon mediante un ANOVA de una vía, con procedimientos post hoc de Tuckey. El nivel a se estableció en $\mathrm{p}$ $<0.05$ para significancia estadística.

Tabla 1

Características básicas de mujeres adultas mayores.

\begin{tabular}{lccc}
\hline & Normo peso & Sobrepeso & Obesidad \\
\hline Sujetos $(\mathrm{n})$ & 8 & 11 & 15 \\
\hline Edad (años) & $74,5 \pm 9,10$ & $71.8 \pm 6.04$ & $74.8 \pm 8.79$ \\
\hline Estatura $(\mathrm{m})$ & $1.50 \pm 4,67$ & $1.49 \pm 3.75$ & $1.47 \pm 5.82$ \\
\hline Peso $(\mathrm{kg})$ & $56,3 \pm 5,02$ & $64.6 \pm 4.55$ & $81.9 \pm 17.7$ \\
\hline IMC $\left(\mathrm{kg} / \mathrm{m}^{-2}\right)$ & $24.7 \pm 1.48$ & $28.8 \pm 1.83$ & $37.7 \pm 6.48$ \\
\hline Grasa corporal $(\%)$ & $31.2 \pm 3.68$ & $39.0 \pm 1.99$ & $48.6 \pm 3.57$ \\
\hline
\end{tabular}

Nota: n: número; m: metros; kg: kilógramos; IMC: índice de masa corporal; \%: porcentaje.

\section{RESULTADOS}

Se observó un mayor tiempo para completar la prueba Timed up and Go en las personas con obesidad a diferencia de las normo peso siendo, estas diferencias entre grupo estadísticamente significativas $\mathrm{p}<0.05$. De igual manera al comparar a las mujeres con sobrepeso con las que presentaban obesidad se observaron diferencias estadísticamente significativas entre estos grupos $\mathrm{p}<0.05$. así mismo, al com- parar las mujeres normo peso con las que presentaban sobrepeso se encontraron diferencias estadísticamente significativas entre estos grupos $\mathrm{p}<0.05$. Además, también se encontraron diferencias estadísticamente significativas entre las variables normo peso, sobre peso y obesidad en la grasa corporal $p<0.05$. Los resultados de estas diferencias en el equilibrio y grasa corporal se presentan en la Tabla 2. 
Tabla 2

Diferencias en el equilibrio dinámico según estado nutricional.

\begin{tabular}{lcccc}
\hline & Normo peso & Sobre peso & Obesidad & $\mathrm{p}$ valor \\
\hline Grasa corporal $(\%)$ & $31.2 \pm 3.68$ & $39.0 \pm 1.99$ & $48.6 \pm 3.57$ & $\mathrm{p}<0.05$ \\
\hline Equilibrio dinámico $(\mathrm{seg})$ & $5.02 \pm 0.52$ & $5.79 \pm 0.55$ & $6.88 \pm 0.71$ & $\mathrm{p}<0.05$ \\
\hline
\end{tabular}

Nota: seg: segundos; \%: porcentaje.

\section{DISCUSIÓN}

Las mujeres adultas mayores que presentaron un exceso en la grasa corporal tanto las con sobrepeso como quienes presentaron obesidad mostraron un mayor deterioro en el equilibrio dinámico al compararlas con las mujeres adultas mayores normo peso, observándose que a mayor grasa corporal mayor era el deterioro en el equilibrio. Estos resultados son similares a los reportados en investigaciones previas como la realizada por Mantello, Hyppolito, Ferriolli, da Costa Lima, \& Moriguti (2012). Estos autores observaron que la obesidad interfirió en el equilibrio de mujeres adultas mayores, al igual que en el estudio de Teasdale et al. (2007) donde se señala que una mayor masa grasa corporal influye en la respuesta del equilibrio en personas mayores, presentándose una mayor inestabilidad postural (Hue et al., 2007; Greve, Bordini, \& Camanho, 2007; Menegoni et al., 2009), lo cual conduce a a un mayor riesgo de caída a esta población envejecida (Teasdale et al., 2007).

Déficits en el sistema propioceptivo, somato sensorial y vestibular ocurren con frecuencia durante el envejecimiento (Clark, Röijezon, \& Treleaven, 2015), donde las personas con obesidad generalmente muestran áreas de contacto plantares más grandes y mayores niveles de presión (Hue et al., 2007; Teasdale et al., 2007). El cuerpo por medio de los pies tiene mecano receptores que reciben sensaciones cutáneas, la obesidad hace que esta respuesta se reduzca, existiendo una adición de los desequilibrios que causan mayor presión en los pies con el suelo lo que reduce la captación de información sensorial (Ghulyan, Paolino, Lopez, Dumitrescu, \& Lacour, 2005). Esto puede ayudar a explicar los resultados observados en el presente estudio.
Dentro de las limitaciones de este estudio están el número de sujetos, por lo cual en futuras investigaciones debería considerarse un tamaño de muestra más grande, de igual manera medir el equilibrio no solamente dinámico si no estático o variables como velocidad de caminata fuerza de agarre entre otras, al igual que la masa grasa, en donde se deben tener indicadores de diversas segmentos del cuerpo como tren superior, inferior, también observar si estos resultados se pueden replicar en hombres adultos mayores.

\section{CONCLUSIÓN}

Las mujeres adultas mayores con obesidad y sobrepeso presentaron un mayor deterioro en el equilibrio dinámico en comparación a las mujeres normo peso lo cual puede afectar negativamente su funcionalidad en este grupo etario. Por lo cual un acumulo excesivo de grasa corporal afecta negativamente la salud de mujeres adultas mayores, pudiendo tener fuertes implicancias en el ámbito clínico de esta población geriátrica. 


\section{REFERENCIAS BIBLIOGRÁFICAS}

Cawthon, P., Fox, K., Gandra, S., Delmonico, M., Chiou, C., Anthony, M., Caserotti, P., et al. (2011). Clustering of strength, physical function, muscle, and adiposity characteristics and risk of disability in older adults. Journal of the American Geriatrics Society, 59(5), 781-7. Recuperado de https://www.ncbi.nlm.nih. gov/pubmed/21568948

Chen, H., \& Guo, X. (2008). Obesity and Functional Disability among Elder Americans. Journal of the American Geriatrics Society, 56(4), 689-694. Recuperado de https://www.ncbi.nlm.nih. gov/pmc/articles/PMC2391089/

Clark, N., Röijezon, U., \& Treleaven, J. (2015). Proprioception in musculoskeletal rehabilitation. Part 2. Clinical assessment and intervention. Manual Therapy, 20(3), 378-387. Recuperado de https://www.ncbi.nlm.nih.gov/pub$\underline{\mathrm{med} / 25787919}$

da Silva Borges, E., de Souza Vale, R., Cader, S., Leal, S., Miguel, F., Pernambuco, C., \& Dantas, E. (2014). Postural balance and falls in elderly nursing home residents enrolled in a ballroom dancing program. Archives of Gerontology and Geriatrics, 59(2), 312-316. Recuperado de https://www.ncbi.nlm.nih.gov/pub$\underline{\mathrm{med} / 24845606}$

Desai, A., Goodman, V., Kapadia, N., Shay, B., \& Szturm, T. (2010). Relationship between dynamic balance measures and functional performance in community-dwelling elderly people. Physical Therapy, 90(5),748-760. Recuperado de https://www.ncbi.nlm.nih.gov/pubmed/20223944
Gallagher, D., Heymsfield, S., Heo, M., Jebb, S., Murgatroyd, P., \& Sakamoto, Y. (2000). Healthy percentage body fat ranges: an approach for developing guidelines based on body mass index. The American Journal of Clinical Nutrition, 72(3), 694-701. Recuperado de https://www. ncbi.nlm.nih.gov/pubmed/10966886

Ghulyan, V., Paolino, M., Lopez, C., Dumitrescu, M., \& Lacour, M. (2005). A new translational platform for evaluating aging or pathology-related postural disorders. Acta Oto-Laryngol, 125: 607-617. Recuperado de https://doi. org/10.1080/00016480510026908

Greve, J., Bordini, A., \& Camanho, G. (2007). Correlation between body mass index and postural balance. Clinics, 62(6), 717-20. Recuperado de https://www. ncbi.nlm.nih.gov/pubmed/18209913

Hue, O., Simoneau, M., Marcotte, J., Berrigan, F., Doré, J., Marceau, P., et al. (2007). Body weight is strong predictor of postural stability. Gait Posture, 26(1), 32-8. Recuperado de https://www.ncbi.nlm. nih.gov/pubmed/16931018

Karimi, M., \& Solomonidis, S. (2011). The relationship between parameters of static and dynamic stability tests. Journal of Research in Medical Sciences, 16(4), 530-535. Recuperado de https:// www.ncbi.nlm.nih.gov/pmc/articles/ $\underline{\text { PMC3214359/ }}$

Lord, S., Ward, J., Williams , P., \& Anstey, K. (1994). Physiological factors associated with falls in older community-dwelling women. Journal of the American Geriatrics Society, 42(10), 1110-1117. Recuperado de https://www.ncbi.nlm.nih. gov/pubmed/7930338 
Mantello, E., Hyppolito, M., Ferriolli, E., da Costa Lima, N., \& Moriguti, J. (2014). Evaluation of the balance of obese and non-obese elderly women by computed dynamic posturography. The Journal of Aging Research \& Clinical Practice, 3(4), 206-210.

Marfell, M., Olds, T., Stewart, A., \& Carte, L. (2006). International standards for anthropometric assessment. South Africa. Editorial: Potchefstroom. Internaional Society for the Advancement of Kinanthropometry. Recuperado de http://www.worldcat.org/title/international-standards-for-anthropometric assessment/oclc/156766813? referer $=\mathrm{di}$ \&ht=edition

Menegoni, F., Galli, M., Tacchini, E., Vismara, L., Cavigioli, M., \& Capodaglio, P. (2009). Gender-specific effect of obesity on balance. Obesity, 17(10), 1951-6. Recuperado de https://www.ncbi.nlm. nih.gov/pubmed/19325540

Mitchell, R., Lord, S., Harvey, L., \& Close, J. (2014). Associations between obesity and overweight and fall risk, health status and quality of life in older people. Australian and New Zealand Journal of Public Health, 38(1), 13-8. Recuperado de https://www.ncbi.nlm.nih.gov/pubmed/24494939

PAR-Q. The Physical Activity Readiness Questionnaire: PAR-Q \& YOU (2002). (accessed www.csep.ca/forms). Disponible en http://uwfitness.uwaterloo.ca/PDF/ par-q.pdf

Posadillo, D., \& Richardson, S. (1991). The timed "Up \& Go": a test of basic functional mobility for frail elderly persons. Journal of the American Geriatrics Society, 39, 142-148. Recuperado de https://www.ncbi.nlm.nih.gov/pub$\underline{\text { med/1991946 }}$
Stenholm, S., Harris, T., Rantanen, T., Visser, M., Kritchevsky, S., \& Ferrucci, L. (2008). Sarcopenic obesity - definition, etiology and consequences. Current Opinion in Clinical Nutrition and Metabolic Care, 11(6), 693-700. Recuperado de https://www.ncbi.nlm.nih.gov/pmc/ articles/PMC2633408/

Teasdale, N., Hue, O., Marcotte, J., Berrigan, F., Simoneau, M., et al. (2007). Reducing weight increases postural stability in obese and morbid obese men. International Journal of Obesity, 31, 153-160. Recuperado de https://www.ncbi.nlm. nih.gov/pubmed/16682978

Tinetti, M. (2003). Clinical practice. Preventing falls in elderly persons. The New England Journal of Medicine, 348(1), 4249. Recuperado de https://www.nejm. org/doi/full/10.1056/NEJMcp020719

Vincent , H., Vincent , K., \& Lamb , K. (2010). Obesity and mobility disability in the older adult. Obesity Reviews, 11(8), 568-79. Recuperado de https://www. ncbi.nlm.nih.gov/pubmed/20059707

Wang, L., Crawford, J., Reppermund, S., Trollor, J., Campbell, L., Baune, B., et al. (2018). Body mass index and waist circumference predict health-related quality of life, but not satisfaction with life, in the elderly. Quality of Life Research, 10.1007. Recuperado de https://www. ncbi.nlm.nih.gov/pubmed/29881897

Zamboni, M., Mazzali, G., Fantin , F., Rossi , A., \& Di Francesco , V. (2008). Sarcopenic obesity: a new category of obesity in the elderly. Nutrition Metabolism \& Cardiovascular Diseases, 18(5), 388-95. Recuperado de https://www.ncbi.nlm. nih.gov/pubmed/18395429 


\section{Dirección para correspondencia}

Jordan Hernández

Profesor de Educación Física

Licenciado en Educación

Técnico Deportivo Universitario

Escuela de Terapia Ocupacional

Universidad Santo Tomás, Osorno, Chile

Los Carrera 753

Código postal 5290000

Contacto:

jordan.eduardo.hernandez@gmail.com

Recibido: 23-10-2018

Aceptado: 06-12-2018 